\title{
Peste des petits ruminants virus non-structural $C$ protein inhibits the induction of interferon- $\beta$ by potentially interacting with MAVS and RIG-I
}

\author{
Li Linjie $^{1,3} \cdot$ Shi Xiaoling ${ }^{2} \cdot$ Ma Xiaoxia ${ }^{1} \cdot$ Cao Xin $^{3} \cdot$ Amjad Ali $^{1} \cdot$ Bai Jialin ${ }^{1}$ (I)
}

Received: 6 August 2020 / Accepted: 10 November 2020 / Published online: 3 January 2021

(C) The Author(s) 2021

\begin{abstract}
Peste des petits ruminants virus (PPRV) causes an acute and highly contagious disease in domestic and wild small ruminants throughout the world, mainly by invoking immunosuppression in its natural hosts. It has been suggested that the non-structural $\mathrm{C}$ protein of PPRV helps in evading host responses but the molecular mechanisms by which it antagonizes the host responses have not been fully characterized. Here, we report the antagonistic effect of PPRV C protein on the expression of interferon- $\beta$ (IFN- $\beta$ ) through both MAVS and RIG-I mediated pathways in vitro. Dual luciferase reporter assay and direct expression of IFN- $\beta$ mRNA analysis indicated that PPRV C significantly down regulates IFN- $\beta$ via its potential interaction with MAVS and RIG-I signaling molecules. Results further indicated that PPRV C protein significantly suppresses endogenous and exogenous IFN- $\beta$-induced anti-viral effects in PPRV, EMCV and SVS infections in vitro. Moreover, PPRV C protein not only down regulates IFN- $\beta$ but also the downstream cytokines of interferon stimulated genes 56 (ISG56), ISG15, C-X-C motif chemokine (CXCL1O) and RIG-I mediated activation of IFN promoter elements of ISRE and NF- $\mathrm{kB}$. Further, this study deciphers that PPRV C protein could significantly inhibit the phosphorylation of STAT1 and interferes with the signal transmission in JAK-STAT signaling pathway. Collectively, this study indicates that PPRV C protein is important for innate immune evasion and disease progression.
\end{abstract}

Keywords Protein $C \cdot$ Interferon- $\beta$ (IFN- $\beta) \cdot$ Luciferase reporter assay $\cdot$ Pest des petits ruminants virus $\cdot$ Antiviral state

\section{Introduction}

Peste des petits ruminants (PPR) is an acute and highly contagious disease of both domestic and wild small ruminants, caused by peste des petits ruminants virus (PPRV), a Morbillivirus of the Paramyxoviridae family [1]. PPR is causing serious economic losses in developing countries

Edited by William Dundon.

Bai Jialin

jlbai@xbmu.edu.cn

1 Key Laboratory of Bioengineering \& Biotechnology of the National Ethnic Affairs Commission, Biomedical Research Center, Northwest Minzu University, Lanzhou 730030, China

2 School of Chemical and Biological Engineering, Lanzhou Jiaotong University, Lanzhou 730070, China

3 College of Life Science and Engineering, Northwest Minzu University, Lanzhou 730030, China where sheep and goats rearing/business make an important component of the livelihood of the poor [2]. Other important members within the Paramyxoviridae family are measles virus $(\mathrm{MeV})$, phocine distemper virus (PDV), rinderpest virus (RPV), cetacean morbillivirus (CeMV), feline morbillivirus (FMV) and canine distemper virus (CDV) [3]. PPRV is a single-stranded, negative-sense, non-segmented RNAvirus with a genome size of up to $15 \mathrm{~Kb}$, having six genes, encoding eight structural and non-structural proteins. Structural proteins are; the fusion protein $(\mathrm{F})$, hemagglutinin membrane glycoprotein $(\mathrm{H})$, large protein $(\mathrm{L})$, matrix protein $(\mathrm{M})$, phosphoprotein $(\mathrm{P})$, and nucleoprotein $(\mathrm{N})$, whereas $\mathrm{W}$ and $\mathrm{C} / \mathrm{V}$ proteins are the non-structural proteins $[4,5]$. Co-transcripts of the $P$ gene as a result of G insertion; single and/or double, at the editing site during transcription, leads to $\mathrm{V}$ and $\mathrm{W}$ proteins synthesis. Likewise, $\mathrm{C}$ protein coding mRNA is also generated from the second ATG codon of the alternate open reading frame (ORF) of the $\mathrm{P}$ cistron [6-8]. During the course of infection and for minimizing host's 
interferon (IFN) responses, both $\mathrm{C}$ and $\mathrm{V}$ proteins are considered to be important [9-12].

Viruses are able to use various strategies to evade the immune responses including the induction of immunosuppression by offsetting host IFN responses, stimulated via pathogen-associated molecular patterns (PAMPs) by cellular pathogen recognition receptors (PRRs). Retinoic acid-inducible gene I (RIG-I)-like receptors (RLR), a PRR, which include important proteins like melanoma differentiation-associated protein 5 (MDA-5), RIG-I, and laboratory of genetics and physiology 2 (LGP2), can detect intracellular pathogens by recognizing specific pathogen-associated RNAs. These receptors contain a DExH/D box RNA helicase domain and a specific carboxy-terminal domain, important for host immunity. Moreover, the two tandem caspase recruitment domains (CARDs) at the amino-termini of MDA-5 and RIG-I are presumed to have a role in the downstream signaling which activates IFN- $\beta$ promoter [13]. LGP2, lacking CARD, on the other hand, activates and represses MDA-5 and RIG-1, respectively [14-16]. LGP2 role in PAMP recognition by MDA-5 and RIG-I has also been suggested [17].

Paramyxovirus $\mathrm{V}$ protein on binding to MDA-5, blocks MDA-5-mediated IFN- $\beta$ induction [18-20]. Dephosphorylation of MDA-5, necessary for MDA-5 downstream signaling applications, has been shown to be blocked by MeV V protein when binds to phosphoprotein phosphatase 1 (PP1) [21]. Likewise, the $\mathrm{V}$ protein of parainfluenza virus type 5 (PIV5) does not interact with RIG-1 [19], but binds to LGP2 $[22,23]$, thereby inhibits IFN- $\beta$ activation by RIG-1 pathway [22]. Moreover, studies indicate that IFN- $\beta$ promoter activation by MDA- 5 can be facilitated by LGP2 whereas PIV5 V may block MDA-5 signals by interacting with LGP2 [22, 24]. There have been three studies reporting that PPRV V protein blocks IFN- $\beta$ induction by binding to MDA-5 [12, $20,25]$. Hence, it is evident that paramyxovirus $\mathrm{V}$ protein plays an important role in the inhibition of IFN- $\beta$ induction by interfering with MDA-5 and RIG-I pathways.

The exact mechanism by which paramyxovirus $C$ proteins interact with IFN- $\beta$ is not clear. C protein of morbillivirus has been shown to play a role in the regulation of viral RNA synthesis, replication, and translation which could lead to suppression of IFN- $\beta$ promoter [26-29]. Engineered viruses, incapable of producing $\mathrm{C}$ proteins, exhibit PAMP in the form of generating more double-stranded RNA (dsRNA) which ultimately activates IFN- $\beta$ promoter via protein kinase $\mathrm{R}$ (PKR) $[30,31]$ and MDA-5 during infection, unlikely wild type viruses [32-34]. It has also been proposed that $C$ protein can block IFN- $\beta$ transcription directly [35] and that IFN- $\beta$ induction by MDA- 5 and RIG-I mediated pathways has not been affected by PPRV, lacking $\mathrm{C}$ gene/protein, albeit they did not find any direct evidence that it blocks either MDA-5 or RIG-I pathways [12]. Therefore, in order to have a deep understanding of the molecular mechanism, we investigated the effect of PPRV C protein on RIG-1 and MAVS mediated induction of IFN- $\beta$, and its downstream activation of other cytokines and its role in PPRV infection.

\section{Materials and methods}

\section{Cells, viruses, antibodies, and reagents}

Human embryonic kidney 293T cells (HEK-293T) and African Green Monkey Kidney Cells (Vero) were grown in DMEM medium (Life Technologies, CA, USA), supplemented with heat-inactivated $10 \%$ fetal bovine serum (FBS; Lanzhou Minhai Biotechnology Co. Ltd, Lanzhou, China), $100 \mathrm{U} / \mathrm{ml}$ penicillin and $100 \mu \mathrm{g} / \mathrm{ml}$ streptomycin (Life Technologies, CA, USA) in a $5 \% \mathrm{CO}_{2}$ incubator at $37^{\circ} \mathrm{C}$. Nigeria $75 / 1$ attenuated vaccine strain of PPRV, purchased from Xinjiang TianKang Animal Husbandry Biotechnology Co. Ltd, was amplified and used to inoculate Vero cells with. Vesicular stomatitis virus (VSV, ATCC VR-1415) and encephalomyocarditis virus (EMCV, GS-01 strain; KJ524643) available at Key Laboratory of Bioengineering and Biotechnology of the National Ethnic Affairs Commission, China, were used in subsequent experiments.

Viral titrations as $50 \%$ tissue culture infectious dose $\left(\mathrm{TCID}_{50}\right)$ from infected vero cells were calculated using Reed-Muench method. Mouse anti-Flag monoclonal antibody (mAb), rabbit anti-HA, anti- $\beta$-Actin and anti-phosphoSTAT1 $\left(\mathrm{Tyr}^{701}\right) \mathrm{mAbs}$, mouse anti-STAT1 mAbs and horse radish peroxidase (HRP)-conjugated sheep anti-mouse IgG and anti-rabbit IgG were purchased from Cell Signaling Technology (USA). TRIzol ${ }^{\mathrm{TM}}$ Reagent (Ambion), OptiMEM (Gibco), and Lipofectamine 2000 (Invitrogen) were purchased from Thermo Fisher Scientific. Dual Luciferase Reporter Gene Assay Kit was purchased from Beyotime Biotechnology (Shanghai, China). Protease and phosphatase inhibitor cocktails were purchased from Roche Pharmaceutical Ltd, Switzerland. ECL substrate luminescence kit (Millipore), PVDF membranes and IFN- $\beta$ were purchased from Sigma-Aldrich, St. Louis, MO, USA.

\section{Plasmids and transfection assays}

pCMV-HA and pMD18-T plasmids were purchased from TaKaRa Medical Biotechnology (Beijing) Co. LTD. pGL3IFN- $\beta$, pGL3-ISRE, pGL3-NF- $\kappa B$, pRL-TK, pRK-FlagRIG-IN, pRK-Flag-MAVS, pRK-Flag-MAD5, pRK-FlagTRAF3, pRK-Flag-TBKI, pRK-Flag-IKKe, pRK-Flag-IRF3, and pRK-Flag-IRF7 were kindly provided by Prof. Qiyun Zhu from Lanzhou Veterinary Research Institute, Chinese Academy of Agricultural Sciences, while expression plasmid, pCMV-HA-C, was constructed in-house. 
HEK-293T cells $\left(2 \times 10^{5}\right)$ in 6 -well plates were cultured (70-80\% confluency) in DMEM and were transfected with pCMV-HA $(2.5 \mu \mathrm{g})$, pCMV-HA-C $(2.5 \mu \mathrm{g})$ and pRK-FlagRIG-IN $(1 \mu \mathrm{g})$ alone, and/or pCMV-HA-C $(1.5 \mu \mathrm{g})$ and pRK-Flag-RIG-IN $(1 \mu \mathrm{g})$ together using Lipofectamine 2000 Transfection Reagent according to the manufacturer's instructions. pCMV-HA was added to ensure cells received equal amount of plasmids in each transfection. At $24 \mathrm{~h}$ post transfection (hpt), cells were harvested for subsequent analysis.

In order to determine the potential interaction between PPRV C protein and endogenous IFNs, the supernatants of respective cultures were harvested at $24 \mathrm{hpt}$ and I $\mathrm{ml}$ of the supernatant was mixed with $1 \mathrm{ml}$ of fresh DMEM medium and were added to fresh HEK-293 cultures and incubated for $24 \mathrm{~h}$, followed by infection with PPRV at 1 multiplicity of infection (MOI), VSV at $0.1 \mathrm{MOI}$ and EMCV at 0.1 MOI.

Similarly, for assessing the interaction between PPRV C protein and exogenous IFNs, the HEK-293 cells $\left(2 \times 10^{5}\right)$, cultured in 6-well plates were transfected with pCMV-HA-C and pCMV-HA for $24 \mathrm{~h}$ and were treated with IFN- $\beta$ (1000 $\mathrm{U} / \mathrm{ml}$ ) for another $24 \mathrm{~h}$, followed by infection with PPRV, VSV and EMCV as discussed earlier. At $24 \mathrm{~h}$ post infection (hpi), cells were harvested for RT-qPCR and western blot analysis.

\section{Quantitative real-time PCR (qPCR)}

Total RNA was extracted from cultures by TRIzol ${ }^{\mathrm{TM}}$ Reagent according to the manufacturer's instructions. First strand cDNA was generated using GoScript ${ }^{\mathrm{TM}}$ Reverse Transcriptase System (Promega) according to the manufacturer's instructions. qPCR was performed with SYBR Green PCR Master Mix (Bio-Rad) on a CFX Connect Real-Time System (Bio-Rad) for the expression of IFN- $\beta, \mathrm{C}-\mathrm{X}-\mathrm{C}$ motif chemokine ligand 10 (CXCL-10), interferon-stimulated genes 56 (ISG56) and ISGI5 and for $L$ of VSV, VPI of EMCV, and $H$ of PPRV. Values obtained for each gene were normalized to that of the gene encoding glyceraldehydephosphate dehydrogenase (GAPDH). Gene specific primers used in this study are listed in Table 1.

\section{Dual luciferase reporter assay}

HEK-293T cells $\left(1 \times 10^{5}\right)$ in 12 -well plates were cultured in DMEM to $70 \sim 80 \%$ confluency and were transfected with internal plasmid, pRL-TK (10 ng) and reporter plasmids, pGL3-IFN- $\beta$ (100 ng), pGL3-ISRE (100 ng), and pGL3-NF- $\mathrm{kB}(100 \mathrm{ng})$ in each well. Then cells were transfected with pCMV-HA (500 ng) and pRK-Flag-RIGIN (100 ng) alone, and with pCMV-HA-C (400 ng) and
Table 1 Gene-specific primers used in the study

\begin{tabular}{ll}
\hline Primer name & Primer sequence (5'-3') \\
\hline IFN- $\beta$-F & 5'-GCTTGGATTCCTACAAAGAAGCA-3' \\
IFN- $\beta$-F & 5'-ATAGATGGTCAATGCGGCGTC-3' \\
ISG56-F & 5'-TCATCAGGTCAAGGATAGTC-3' \\
ISG56-R & 5'-CACACTGTATTTGGTGTCTAGG-3' \\
ISG15-F & 5'-AGGACAGGGTCCCCCTTGCC-3' \\
ISG15-R & 5'-CCTCCAGCCCGCTCACTTGC-3' \\
CXCL10-F & 5'-GTGGCATTCAAGGAGTACCTC-3' \\
CXCL10-R & 5'-TGATGGCCTTCGATTCTGGATT-3' \\
H of PPRV-F & 5'-CTGAATACCAACATTGAG-3' \\
H of PPRV-R & 5'-GAGGAACTTAATCTTATCG-3' \\
VP1 of EMCV-F & 5'-GCTGTCTGGTATAATGGA-3' \\
VP1 of EMCV-R & 5'-TGATACAGTACAATTSTTTTGGGAC-3' \\
L of VSV-F & 5'-GAGACTTTCTGTTACGGGATCTGG-3' \\
L of VSV-R & 5'-TTGCGGCCGCATGTCAACAAGGGG \\
GAPDH-F & CTGGAATGTAT-3' \\
GAPDH-R & 5'-CACAAGCTTCCCGTTCTCAG-3' \\
\hline
\end{tabular}

pRK-Flag-RIG-IN (100 ng) together using Lipofectamine 2000 Transfection Reagent according to the manufacturer's instructions. Empty vectors were added to ensure cells received equal amount of plasmids in each transfection. At $24 \mathrm{hpt}$, cell lysates were prepared and analyzed for Firefly and Renilla luciferase activity using a dual-luciferase reporter assay system (GLoMax 20/20, Promega, USA), according to the manufacturer's protocol.

For screening other components of the RIG-I mediated pathway, HEK-293T cells in 12-well plates were transfected with internal plasmid, pRL-TK (10 ng) and reporter gene plasmids in each well. Then cells were transfected with pGL3-IFN- $\beta$ (100 ng) and pRK-Flag-RIG-IN (100 ng), pRK-Flag-MAVS (100 ng), pRK-Flag-IKK $\varepsilon$ (100 ng), pRK-Flag-TBKI (100 ng), pRK-Flag-TRAF3 (100 ng), pRK-Flag-IRF3 (100 ng), pRK-Flag-IRF7 (100 ng) alone and/or together with pCMV-HA-C (400 ng) in each well. Empty vectors were added to ensure cells received equal amount of plasmids in each transfection. At $24 \mathrm{hpt}$, cell lysates were prepared and analyzed for Firefly and Renilla luciferase activity using a dual-luciferase reporter assay kit.

\section{Western blotting}

Cells were washed with phosphate-buffered saline (PBS) and lysed using NP40 cell lysis buffer (20 mM Tris-HCl, $150 \mathrm{mM} \mathrm{NaCl}, 1 \mathrm{mM}$ EDTA, 1\% Nonidet P-40) supplemented with a protease and phosphatase inhibitor cocktails for $30 \mathrm{~min}$ at $4^{\circ} \mathrm{C}$. Cells lysates were subjected to $10 \%$ 
SDS-PAGE electrophoresis and were subsequently transferred to a PVDF membrane. Membranes were blocked by $5 \%$ skimmed milk for $1 \mathrm{~h}$, followed by incubation with appropriate antibodies at $4^{\circ} \mathrm{C}$ overnight. The membranes were exposed to chemiluminescence reagents as described previously [36]. Mouse anti-Flag mAb, rabbit anti-HA mAb, rabbit anti-phospho-STAT1 $\left(\mathrm{Tyr}^{701}\right) \mathrm{mAb}$ and mouse anti-STAT1 $1 \mathrm{mAb}$ were used to detect the expression of RIG-IN, HA, total STAT1, and phosphorylated STAT1, respectively. $\beta$-actin was used as a loading control.

\section{Statistical analysis}

Statistical analysis was performed using GraphPad Prism version 5.0 (GraphPad Software, San Diego, CA, USA). Data is presented as mean \pm SEM with an error bar that represents at least three independent experiments. Differences in indicators between treatment groups and controls were assessed using Student's $t$-test. A two-tailed test with $p$ value $<0.05$ corresponds to statistically significant difference and is marked as "*" while $p<0.01$ and $p<0.001$ are marked as "**" and "***" respectively, indicating higher significant differences.

\section{Results}

\section{PPRV C protein antagonizes INF- $\beta$ and its downstream cytokines induced by RIG-I in RLR signaling pathway}

Initially, western blot (WB) was performed to determine the expressions of PPRV C and RIG-I proteins when pCMV-HA-C and pRK-Flag-RIG-IN were co-transfected into HEK-293T cells (Fig. 1a). The qPCR analysis indicated that PPRV C significantly $(p<0.001)$ down-regulated IFN- $\beta, I S G 56, I S G 15$ and $C X C L 10$, induced by RIGIN (Fig. $1 \mathrm{~b}$ to e).

\section{PPRV C protein hampers type I interferon response in vitro}

Dual luciferase reporter assay was performed to detect whether PPRV $C$ protein affects the activities of IFN- $\beta$ and its downstream IFN stimulated response element (ISRE) and nuclear factor kappa-B $(\mathrm{NF}-\kappa \mathrm{B})$. The relative luciferase activity of reporter genes of IFN- $\beta, I S R E$ and $N F-\kappa B$ observed in co-transfected pRK-Flag-RIG-IN and pCMV-HA-C cells was significantly less than in transfected
pRK-Flag-RIG-IN cells as indicated in Fig. 2; panels a $(p<0.001), \mathrm{b}(p<0.01)$, and $\mathrm{c}(p<0.001)$, respectively. This indicates that $\mathrm{C}$ protein of PPRV checks RIG-I mediated IFN- $\beta$ at transcriptional level and antagonizes antiviral response.

\section{Screening of the target molecules in RLR signaling pathway}

To evaluate whether PPRV C protein interacts with RIG-I, mitochondrial antiviral signaling (MAVS), I-kappa kinase (IKK $\varepsilon$ ), TRAF family member-associated NF- $\kappa \mathrm{B}$ activator (TANK)-binding kinase 1 (TBKI), tumor necrosis factor receptor associated factor 3 (TRAF3), interferon regulatory factor 3 (IRF3), and IRF7, dual luciferase reporter gene assays were carried out using RIG-I responsive reporters pRK-Flag-RIG-IN, pRK-Flag-MAVS, pRK-Flag-IKK $\varepsilon$, pRK-Flag-TBKI, pRK-Flag-TRAF3, pRK-Flag-IRF3, and pRK-Flag-IRF7, respectively (Fig. 3). Expression of IFN$\beta$ responsive promoter under the influence of $\mathrm{C}$ protein was significantly lower than activated by RIG-IN (Fig. 3a, $p<0.001$ ) and by MAVS (Fig. 3b, $p<0.001$ ). On the other hand, the effect of the $\mathrm{C}$ protein on the expressions of $I K K \varepsilon$, TBKI, TRAF3, IRF3, and IRF7 was not significant ( $p>0.05)$ (Fig. 3c, d, e, f, and g).

In order to evaluate whether IFN- $\beta$ suppression by $\mathrm{C}$ protein is dose-dependent, HEK-293T cells were transfected with gradient doses of pCMV-HA-C (100, 250 or $400 \mathrm{ng})$ against a constant concentration of pRK-Flag-RIG-IN (100 ng) or pRK-Flag-MAVS (100 ng) for $24 \mathrm{~h}$ and were analyzed. Analysis indicated that with the increase in concentration of pCMV-HA-C from 100 to $400 \mathrm{ng}$, the expression of RIG-I mediated $I F N-\beta$ diminished significantly (Fig. 3h). Similarly, results demonstrated that $\mathrm{C}$ protein significantly $(p<0.001)$ inhibits MAVS-mediated IFN- $\beta$ promoter activation in a dose dependent manner as shown in Fig. $3 i$.

\section{PPRV C protein promotes virus replication via decreasing the endogenous and exogenous IFN responses}

As the expression of RIG-IN can in turn induce the expression of IFN- $\beta$, therefore, the supernatant of the overexpressed RIG-IN cells was taken as the source of endogenous IFNs, and will be referred to as conditioned medium. The expression of $H$ gene of PPRV in the co-transfected RIG-IN and HA-C was significantly higher $(p<0.001)$ than that in RIG-IN cells at 24 hpi (Fig. 4a). The expression profiles of VPl gene of EMCV and $L$ of VSV in co-transfected cells at 24 hpi paralleled that of the $H$ expression of PPRV 
Fig. 1 PPRV C protein inhibits the gene expression of INF- $\beta$ and its downstream factors induced by RIG-IN at transcriptional level. HEK-293T cells $\left(2 \times 10^{5}\right)$ in 6 -well plates were cultured (70-80\% confluency) and were transfected with pCMV-HA $(2.5 \mu \mathrm{g})$, pCMV-HA-C (2.5 $\mu \mathrm{g})$, pRKFlag-RIG-IN $(1 \mu \mathrm{g})$ alone, and co-transfected with pCMV-HA$\mathrm{C}(1.5 \mu \mathrm{g})$ and pRK-Flag-RIGIN $(1 \mu \mathrm{g})$ using Lipofectamine 2000 Transfection Reagent. At $24 \mathrm{~h}$ post transfection (hpt), the expression of Flag-RIGIN and HA-C was detected by western blotting with antiFlag $\mathrm{mAb}$ and anti-HA mAb, respectively. The expression of $\beta$-actin was used as a loading control. The mRNA expression of each IFN- $\beta$, ISG56, ISG15 and $C X C L-10$ was measured by RT-qPCR. a Western blotting has shown that HA-C and Flag-RIG-IN expressed in the transfected cells. b The mRNA expression of $I F N-\beta$ has been reduced to almost half under the effect of PPRV C as compared to RIG-1N. Similarly, mRNA expression of ISG56 (c) ISG15 (d) and $C X C L-10$ (e) have been down-regulated by $P P R V C$ expression

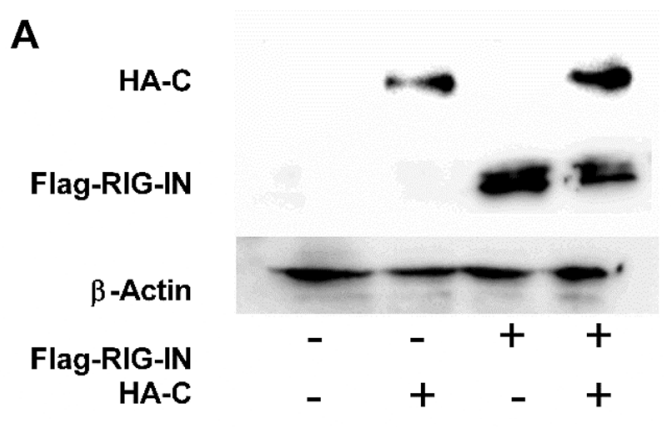

B

IFN- $\beta$

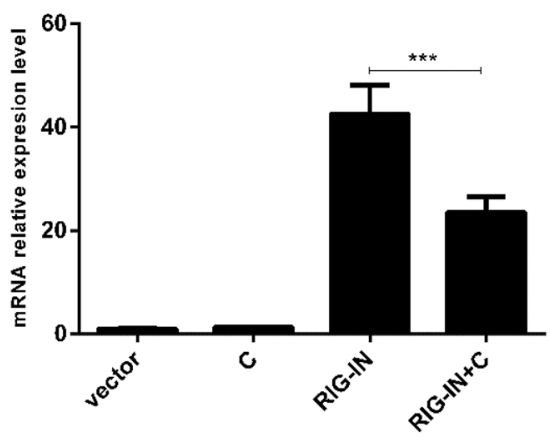

D

ISG15

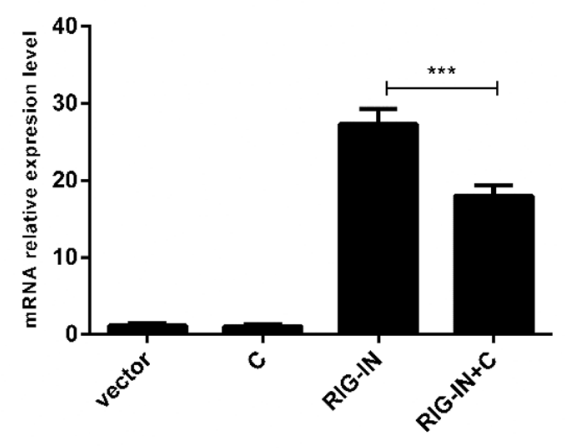

E

C

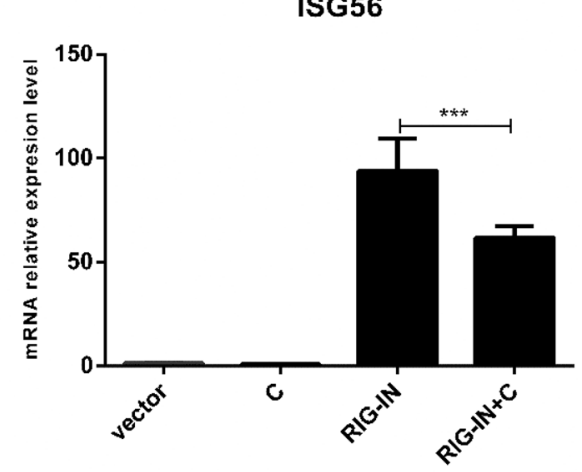

CXCL-10

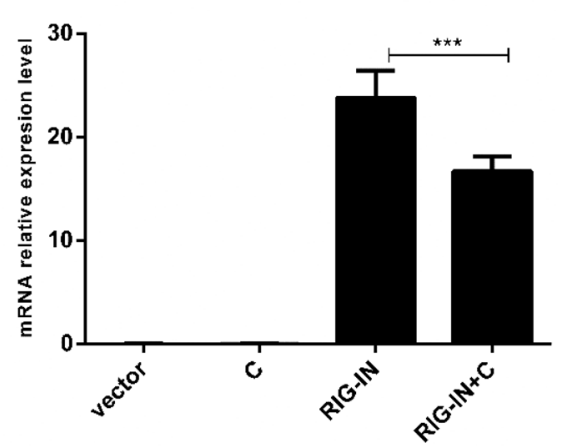

(Fig. 4b and c). Moreover, the expression of $H$ gene of PPRV in the transfected HA-C cells treated with IFN- $\beta$ (exogenous) was significantly higher $(p<0.01)$ than that in transfected HA cells at 24 hpi (Fig. 5a). Similarly, the expression of both $V P 1$ and $L$ genes of EMCV and VSV, respectively in HA-C transfected cells treated with IFN- $\beta$ were significantly higher $(p<0.001)$ than that in HA transfected cells at $24 \mathrm{hpi}$ (Fig. $5 \mathrm{~b}$ and c). C protein expression as evident by western blot analysis has also been shown in Fig. 5.

\section{PPRV C protein inhibits the phosphorylation of STAT1}

To investigate the potential role of PPRV C protein in causing immunosuppression of the host via JAK-STAT pathway, HEK-293T cells were transfected with pCMV-HA-C and pCMV-HA separately and together, and then treated with INF- $\beta$ for $24 \mathrm{~h}$. Results indicated that the expression of total STAT1 in transfected cells remained unchanged, irrespective of the presence or absence of PPRV C protein (Fig. 6) but 

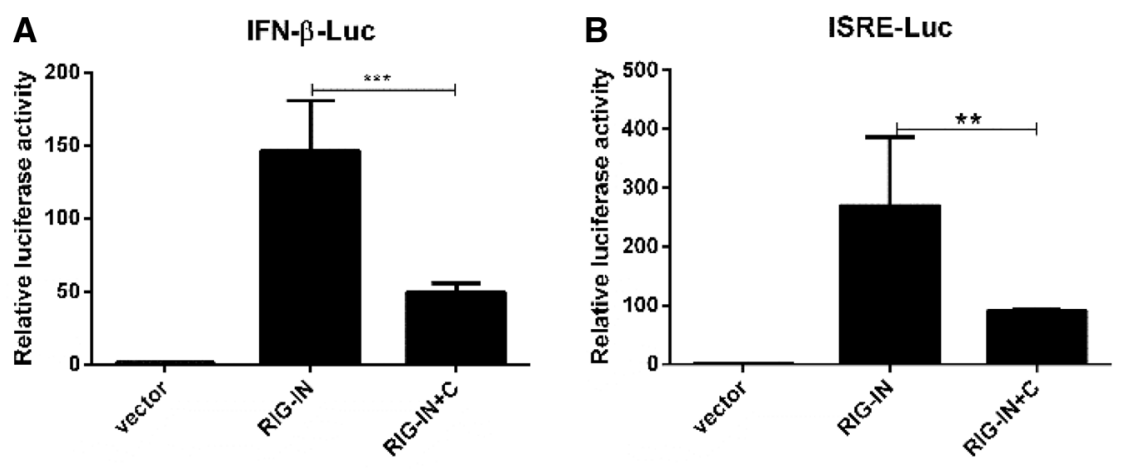

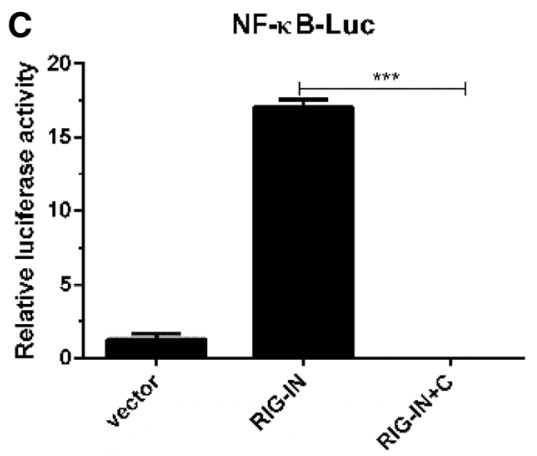

Fig. 2 PPRV C protein inhibits RIG-IN mediated reporter gene activity. HEK-293T cells $\left(1 \times 10^{5}\right)$ in 12 -well plates were cultured $(70$ $80 \%$ confluency) and were transfected with internal plasmid pRLTK (10 ng) along with reporter plasmids pGL3-IFN- $\beta$ (100 ng) (a), pGL3-ISRE (100 ng) (b) and pGL3-NF-кB (100 ng) (c) in each well, respectively. Then cells were transfected with pCMV-HA (500 ng), pCMV-HA-C (400 ng) and pRK-Flag-RIG-IN (100 ng) alone, and with pCMV-HA-C (400 ng) and pRK-Flag-RIG-IN (100 ng) together

the phosphorylation of STAT1 was enhanced when PPRV $\mathrm{C}$ was not expressed and decreased when PPRV C was expressed after IFN- $\beta$ treatment (Fig. 6).

\section{Discussion}

PPRV is one of the major problems of the sheep and goat industries worldwide that inflicts severe economic losses due to high morbidity and mortality rates. Genome of the PPRV expresses $\mathrm{C}$ protein, which is a non-structural protein of an alternate open reading frame of $P$ cistron and has been thought to be important for bringing about infections [8, 37]. Studies had revealed that $C$ protein though not phosphorylates but binds to the $\mathrm{L}$ protein and localizes in the nucleus and cytoplasm [38], thereby contribute to immune evasion and pathogenicity [39-41]. For C protein, in case of Morbillivirus, two mechanisms have been recognized which are responsible for halting IFNs production; by restricting viral replication, thereby decreasing the amount of motifs that PRRs recognizes [28, 29, 42] and direct interference in the PRRs signaling that leads to the inhibition of IFNs transcription [35, 43, 44]. It has been reported that MeV-C and RPV-C proteins of the Morbilliviruses interact with IRF3 in the nucleus and thereby block the activation of IFN-I, though the exact mechanism is not fully known [35, 43]. IRF3-dependent transcription is disrupted by $\mathrm{C}$ protein and does not interact with the phosphorylation of IRF3, its dimerization or accumulation [35], indicating that its underlying mechanism is dependent on steric hindrance of IRF3 dimers with the IFN- $\beta$ promoter. Moreover, it has using Lipofectamine 2000 Transfection Reagent. Empty vectors were added to ensure cells received equal amount of plasmids in each transfection. At $24 \mathrm{hpt}$, cell lysates were analyzed for Firefly and Renilla luciferase activity using a dual-luciferase reporter assay kits. The expression levels of IFN- $\beta$ (a), ISRE (b) and $N F-\kappa B$ (c) were significantly reduced in pCMV-HA-C and pRK-Flag-RIG-IN co-transfected cells than that of pRK-Flag-RIG-IN transfected cells

been observed that $\mathrm{MeV}-\mathrm{C}$ and $\mathrm{V}$ proteins can also block NF-kB pathway, highlighting its vast role in immune suppression and disease progression [44]. Studies also indicate that though $\mathrm{V}$ protein is the principal IFNs signaling antagonist during infection [29], MeV-C can also block it [45]. It has also been reported that PPRV lacking $C$ protein did not hamper host IFN- $\beta$ response [12] but its direct effect was not elucidated.

The exact mechanism by which PPRV evades the immune responses and initiates infection in relation to the $\mathrm{C}$ protein is poorly understood. Therefore, we carried out this study in detail to unveil the potential role of PPRV C protein in antagonizing host responses and causing infection by employing HEK-293T cells which is a commonly used, amenable and efficient model system for infection and protein expression studies [46, 47]. Nevertheless, it is a human cell line and so it should be pointed out that it may not be an ideal model for the study of a small ruminant infecting viruses like PPRV.

Here, we report that PPRV C protein significantly antagonizes the expression of $I F N-\beta$ (Fig. 1b) and also interferes with RIG-IN mediated expression of ISG56, ISG15 and CXCL-10 in vitro as shown in Fig. 1, panels C, D and E, respectively. Dual luciferase assay indicated that $C$ protein of PPRV significantly suppresses $I F N-\beta, I S R E$ and $N F-\kappa B$ at the transcriptional level (Fig. 2).

Analysis of the target molecules in the RLR pathway indicated that $C$ protein potentially interacts with RIG-I and MAVS (Fig. 3a and b) as compared to the rest of the molecules (Fig. $3 \mathrm{c}$ to g) and downregulates their expression substantially that in turn check the IFN $-\beta$ response. To peruse 
Fig. 3 Effect of the expression of the seven possible target molecules of PPRV C proteininduced activation of $I F N-\beta$ promoter in RLR pathway. Monolayer HEK-293T cells in 12-well plates were transfected with internal reference gene plasmid pRL-TK (10 ng) and reporter gene plasmid pGL3IFN- $\beta$ (100 ng) in each well. Cells were then transfected with pRK-Flag-RIG-IN (100 ng), pRK-Flag-MAVS (100 ng), pRK-Flag-IKKe (100 ng), pRK-Flag-TBKI (100 ng), pRK-Flag-TRAF3 (100 ng), pRK-Flag-IRF3 (100 ng), and pRK-Flag-IRF7 (100 ng) alone and together with pCMV-HA-C $(400 \mathrm{ng})$ in each well. For in depth analysis, various doses of pCMV-HA-C (100, 250 or $400 \mathrm{ng}$ ) against constant values of either pRK-Flag-RIG-IN $(100 \mathrm{ng})$ or pRK-Flag-MAVS (100 ng) were used (h and i). Empty vectors were added to ensure cells received equal amount of plasmids in each transfection. At $24 \mathrm{hpt}$, cell lysates were analyzed for Firefly and Renilla luciferase activity using a dual-luciferase reporter assay kit. Expression of IFN- $\beta$ responsive promoter under the effect of $\mathrm{C}$ protein was significantly lower than activated by RIG-I (a) and by MAVS (b). On the contrary, the effect of $C$ protein on the expression levels of $I K K \varepsilon(\mathbf{c}), T B K I(\mathbf{d}), T R A F 3$ (e), $I R F 3$ (f), and IRF7 (g) was not significant. h RIG-I-mediated $I F N-\beta$ and MAVS-mediated IFN- $\beta$ promoter activation were found to be significantly inhibited by $\mathrm{C}$ protein (h and i respectively) and were dose dependent; increase in dose from 100 to $400 \mathrm{ng}$ halted IFN- $\beta$ expression in each case
A

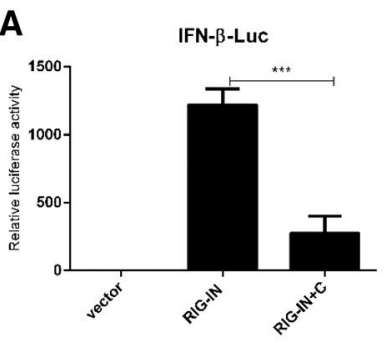

C

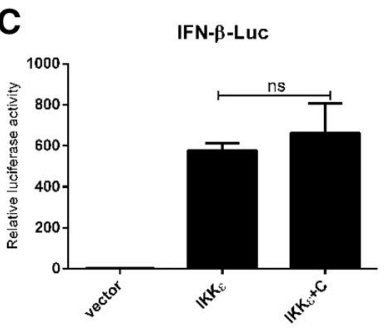

E

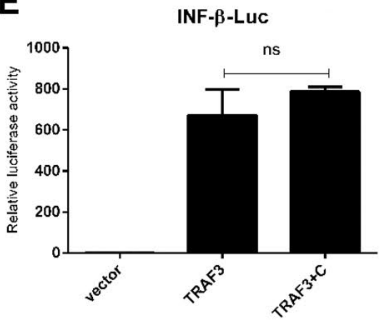

G

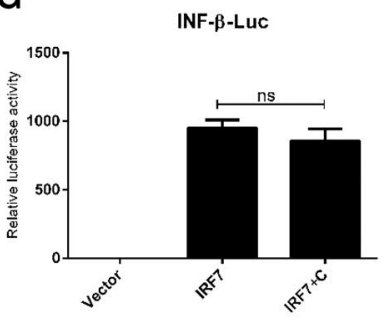

H

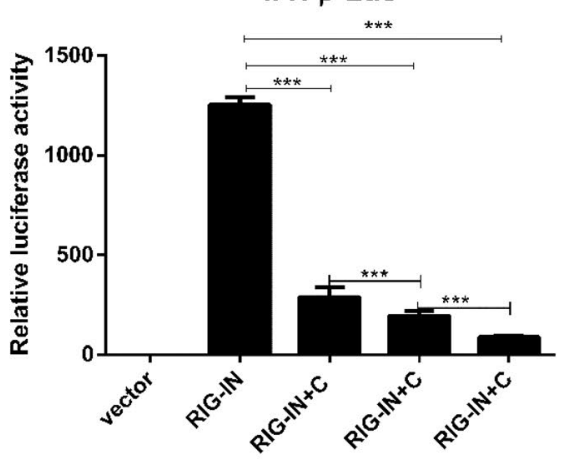

RIG-IN(ng) $\quad 0 \quad 100 \quad 100 \quad 100 \quad 100$
B

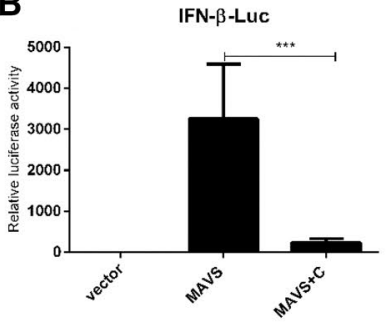

D

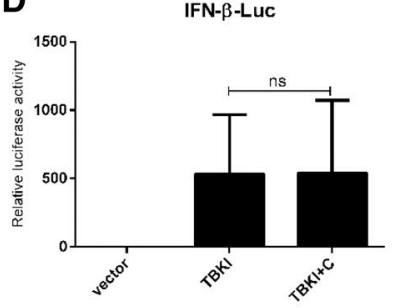

F

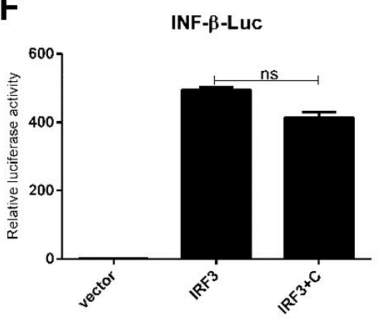

I

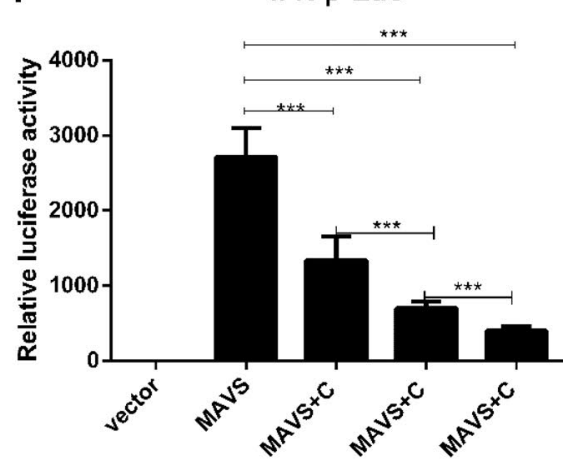

$\begin{array}{llllll}\text { MAVS(ng) } & 0 & 100 & 100 & 100 & 100\end{array}$ 
A

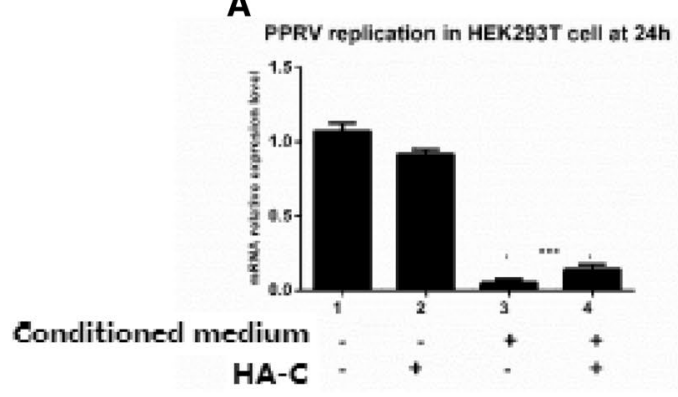

B

EMCV replication in HEK293T cell at $24 h$

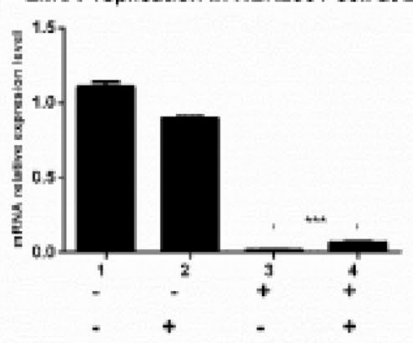

C

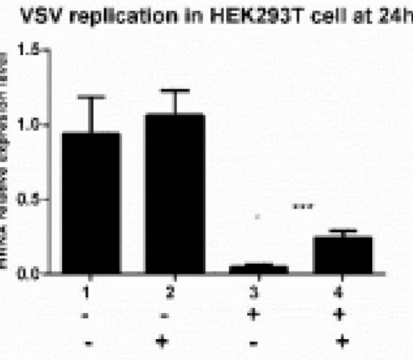

Fig. 4 PPRV C protein inhibits the anti-viral effect via decreasing the production of endogenous IFN response. HEK-293T cells $\left(2 \times 10^{5}\right)$ in 6-well culture plates were cultured in DMEM supplemented with $10 \%$ FBS for growing to $70-80 \%$ confluency. Cells were transfected with pCMV-HA $(2.5 \mu \mathrm{g})$, pCMV-HA-C $(2.5 \mu \mathrm{g})$ and pRK-Flag-RIGIN $(1 \mu \mathrm{g})$ alone, and pCMV-HA-C $(1.5 \mu \mathrm{g})$ and pRK-Flag-RIG-IN $(1 \mu \mathrm{g})$ together using Lipofectamine 2000 Transfection Reagent according to the manufacturer's instructions. Empty vectors were added to ensure cells received equal amount of plasmids in each transfection. At $24 \mathrm{hpt}$, the supernatants were harvested and then $1 \mathrm{ml}$ supernatants and $1 \mathrm{ml}$ fresh DMEM medium were added to the new
HEK-293T cells and cultured for $24 \mathrm{~h}$, followed by infection with 1 MOI PPRV, 0.1 MOI VSV and 0.1 MOI EMCV, respectively. At $24 \mathrm{~h}$ post infection (hpi), all infected cells were harvested for RT-qPCR to measure the gene expression of PPRV H,EMCV VPI and VSV L. a The expression of PPRV $H$ gene in RIG-IN and PPRV C co-transfected cells was most significantly higher than that in RIG-IN transfected cells $(p<0.001)$; b The expression of EMCV VP1 gene in RIGIN and PPRV C co-transfected cells was most significantly higher than that in RIG-IN transfected cells $(p<0.001)$; $\mathbf{c}$ The expression of VSV L gene in RIG-IN and PPRV C co-transfected cells was most significantly higher than that in RIG-IN transfected cells $(p<0.001)$

\section{A}

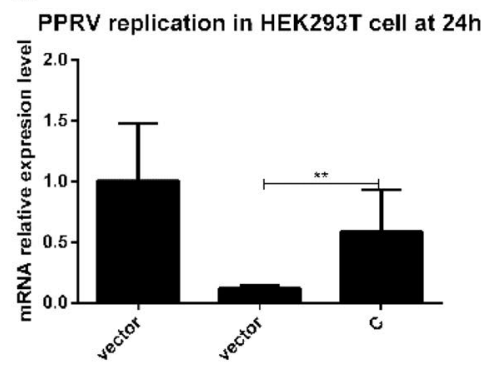

IFN- $\beta$

\section{B}
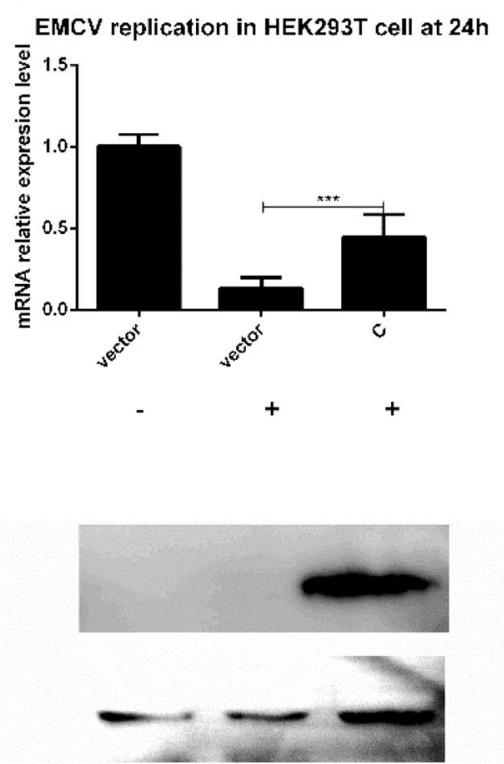

\section{C}
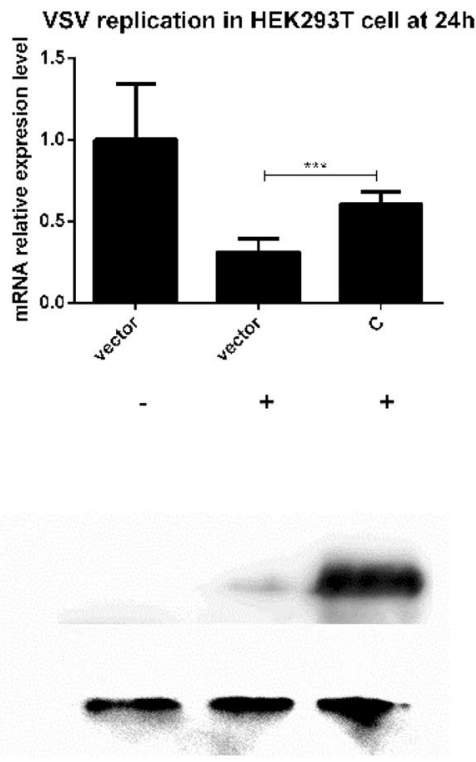

Fig. 5 PPRV C protein inhibits the anti-viral effect via decreasing the production of exogenous IFN response. Monolayer HEK-293T cells in 6 well culture plates were transfected with pCMV-HA $(2.5 \mu \mathrm{g})$ or pCMV-HA-C $(2.5 \mu \mathrm{g})$. At $24 \mathrm{hpt}$, these cells were treated with IFN- $\beta$ $(1000 \mathrm{U} / \mathrm{ml})$ for $24 \mathrm{~h}$ and then followed by infection with PPRV (1 MOI), VSV (0.1 MOI) and EMCV (0.1 MOI). At $24 \mathrm{hpi}$, all infected cells were harvested for RT-qPCR to measure the gene expression of
$P P R V H, E M C V V P 1$, and $V S V L$. a The expression of $P P R V H$ in the transfected HA-C cells treated with IFN- $\beta$ was significantly higher than that in transfected HA cells $(p<0.01)$; b The expression of $E M C V V P 1$ in the transfected HA-C cells treated with IFN- $\beta$ was significantly higher than that in transfected HA cells $(p<0.001)$; $\mathbf{c}$ The expression of $V S V L$ in the transfected HA-C cells treated with IFN- $\beta$ was significantly higher than that in transfected HA cells $(p<0.001)$ 


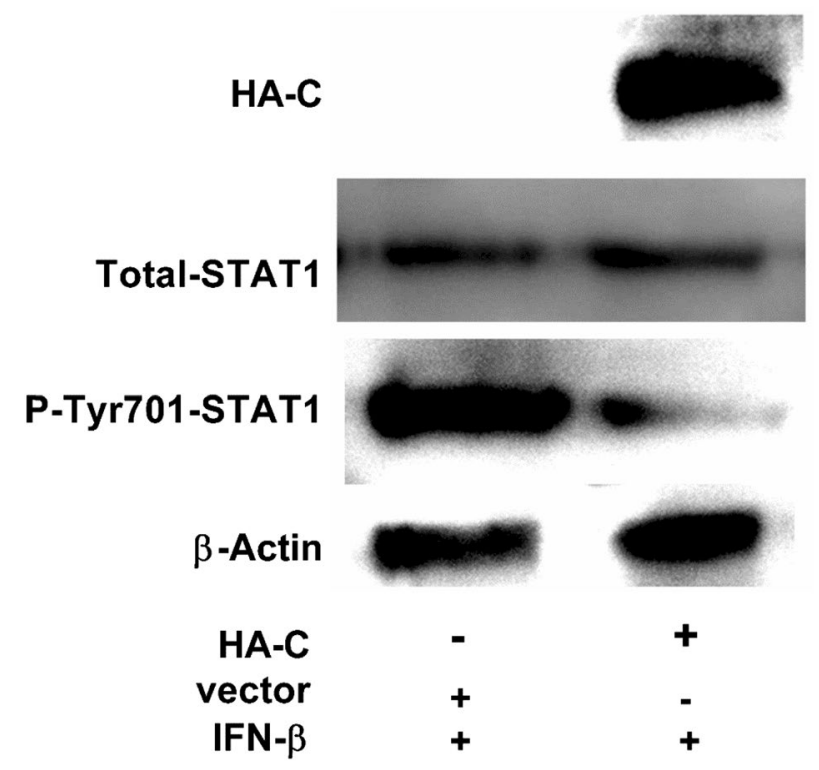

Fig. 6 PPRV C protein inhibits the phosphorylation of STAT1. Monolayer HEK-293T cells in 6-well culture plates were transfected with pCMV-HA $(2.5 \mu \mathrm{g})$ and pCMV-HA-C $(2.5 \mu \mathrm{g})$, respectively. At 24 hpt, these cells were treated with IFN- $\beta(1000 \mathrm{U} / \mathrm{ml})$. At $24 \mathrm{~h}$ post treatment, cells were processed for the expression of total STAT1 and phosphorylated STAT1 with mouse anti-STAT1 mAb and rabbit antiphospho-STAT1 $\left(\mathrm{Tyr}^{701}\right) \mathrm{mAb}$, respectively. Phosphorylated STAT1 expression was inhibited when $\mathrm{C}$ protein was expressed, with no effect on total STAT1. $\beta$-actin was used as a loading control. A representative image of the three independent experiments is shown here

this interaction further, gradient expression of the $\mathrm{C}$ protein was employed. The analysis indicated that a higher expression of C significantly halted the expression of RIG-IN and MAVS-mediated $I F N-\beta$ in a dose dependent manner (Fig. $3 \mathrm{~h}$ and $\mathrm{i}$ ). This indicates that $\mathrm{C}$ protein plays an important role in counteracting the innate responses which leads to disease progression.

This study further communicates that $\mathrm{C}$ protein promotes the replication of PPRV, EMCV and VSV significantly in vitro by halting endogenous IFNs (Fig. 4a, $\mathrm{b}$ and c) which implies its importance and a universal role in infection and disease progression. IFN- $\beta$ as a source of exogenous IFNs was supplied to respective cultures in order to gauge the effect of the $\mathrm{C}$ protein with. Analysis revealed that $\mathrm{C}$ protein significantly hampered the effect of exogenous IFNs and promoted the replication of PPRV, EMCV, and VSV as indicated in Fig. 5, panels a, b, and $\mathrm{c}$, respectively which highlights the crucial role of the $\mathrm{C}$ protein in causing infection and immune evasion. The results of the current study are congruent with previous studies on the $\mathrm{C}$ proteins of other viruses. Studies indicated that Morbillivirus RPV C protein inhibits the activation of the IFN- $\beta$ promoter, and blocking $\mathrm{C}$ protein in the recombinant RPV led to much greater expression of IFN- $\beta$ promoter [43]. A similar mechanism of Morbillivirus $\mathrm{C}$ protein in a recombinant $\mathrm{MV}$, lacking $\mathrm{C}$ expression has previously indicated an increase in IFNs induction [28]. Studies on MV or RPV with knockout C, which rendered reduced growth in interferon-producing cells than $\mathrm{V}$ knockout equivalent viruses were also been reported previously [26, 48, 49].

Furthermore, this study finds that PPRV C protein can also inhibit the phosphorylation of STAT1, as revealed by three independent experiments (Fig. 6) and thereby hinders the signal transmission in JAK-STAT signaling pathway and impairs the host antiviral responses.

In summary, this study communicates a comprehensive report on the role of the C protein of PPRV in immune evasion and diseases progression via offsetting endogenous and exogenous IFN responses by potentially interacting with RIG-1 and MAVS and by inhibiting the phosphorylation of STAT1 which disrupts the JAK-STAT signaling pathway in vitro (Fig. 7). Our findings emphasize on the $\mathrm{C}$ protein as a potential vaccine and therapeutic target and warrant further study in the same domain. 


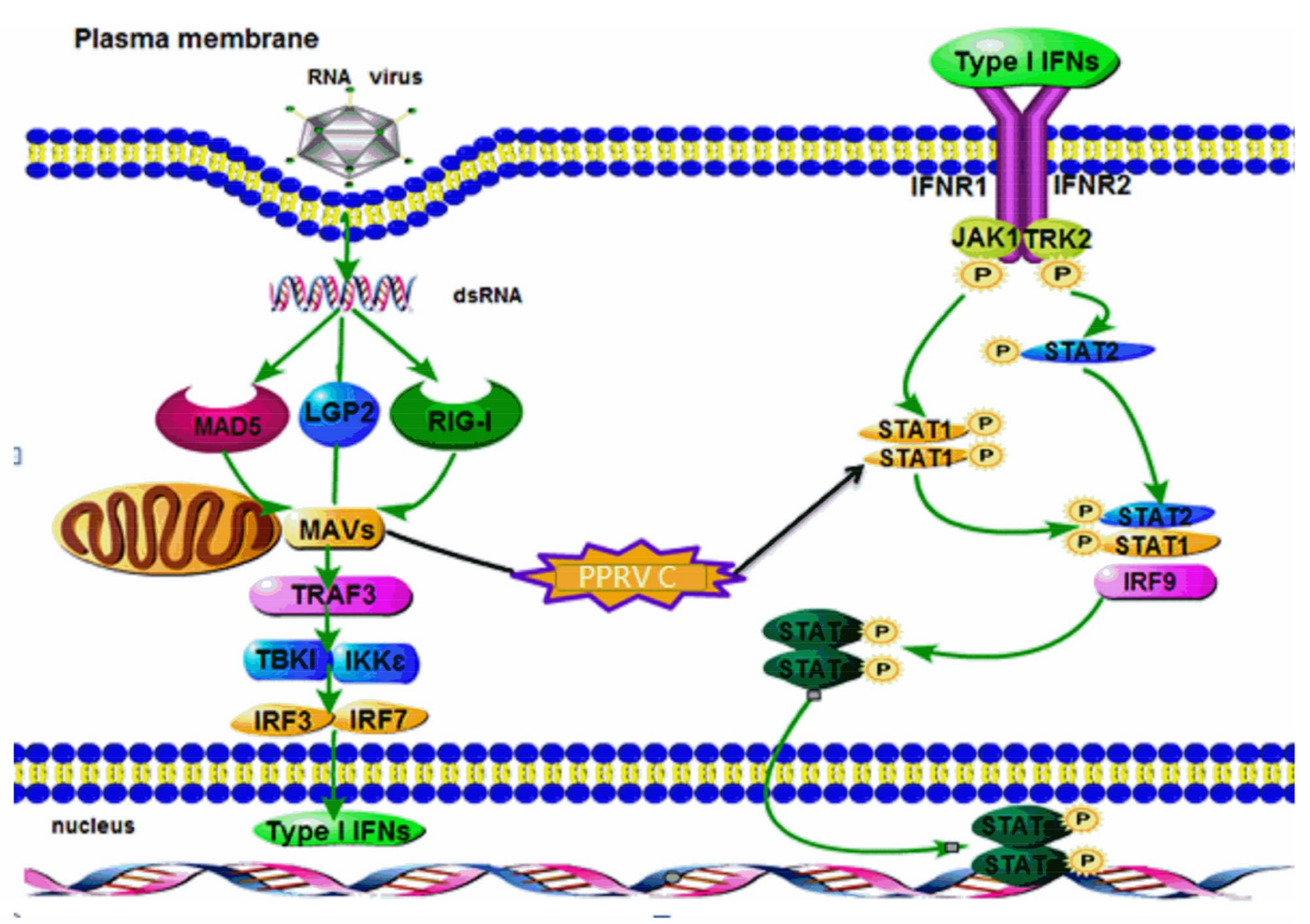

Fig. 7 Mechanistic illustration of the interaction of PPRV C protein. General mechanism of PPRV entry, activation of various pathways, MAVS, JAK-STAT and IFN- $\beta$ induction among others have been shown

Acknowledgements We thank Prof. Qiyun Zhu from Lanzhou Veterinary Research Institute, Chinese Academy of Agricultural Sciences (CAAS) for kindly providing plasmids.

Author contributions Conceived and designed the experiments: BJL CX MXX. Performed the experiments: LLJ SXL. Analyzed the data: LLJ BJL SXL. Wrote the paper: BJL LLJ AA.

Funding This work was supported by the National Natural Science Foundation of China (31860696), the Fundamental Research Funds for the Central Universities (31920190003) and the Changjiang Scholars and Innovative Research Team in the University (IRT_17R88).

Data availability The original data supporting the conclusions of this manuscript will be provided by the authors to any qualified researcher without reservation.

\section{Compliance with ethical standards}

Animal rights Animals were not involved in this study.

Conflict of interest The authors declare that the research was conducted in the absence of any commercial or financial relationships that could be construed as a potential conflict of interest.
Consent for publication Yes.

Open Access This article is licensed under a Creative Commons Attribution 4.0 International License, which permits use, sharing, adaptation, distribution and reproduction in any medium or format, as long as you give appropriate credit to the original author(s) and the source, provide a link to the Creative Commons licence, and indicate if changes were made. The images or other third party material in this article are included in the article's Creative Commons licence, unless indicated otherwise in a credit line to the material. If material is not included in the article's Creative Commons licence and your intended use is not permitted by statutory regulation or exceeds the permitted use, you will need to obtain permission directly from the copyright holder. To view a copy of this licence, visit http://creativecommons.org/licenses/by/4.0/.

\section{References}

1. Aguilar XF, Fine AE, Pruvot M, Njeumi F, Walzer C, Kock R, Shiilegdamba E (2018) PPR virus threatens wildlife conservation. Science 362:165-166

2. Parida S, Muniraju M, Mahapatra M, Muthuchelvan D, Buczkowski H, Banyard AC (2015) Peste des petits ruminants. Vet Microbiol 181:90-106 
3. Woo PCY, Lau SKP, Wong BHL, Fan RYY, Wong AYP, Zhang AJX, Wu Y, Choi GKY, Li KSM, Hui J, Wang M, Zheng BJ, Chan KH, Yuen KY (2012) Feline morbillivirus, a previously undescribed paramyxovirus associated with tubulointerstitial nephritis in domestic cats. Proc Natl Acad Sci USA 109:5435-5440

4. Bellini WJ, Englund G, Rozenblatt S, Arnheiter H, Richardson CD (1985) Measles virus P gene codes for two proteins. J Virol 53:908-919

5. Cattaneo R, Kaelin K, Baczko K, Billeter MA (1989) Measles virus editing provides an additional cysteine-rich protein. Cell 56(5):759-764

6. Hausmann S, Garcin D, Delenda C, Kolakofsky D (1999) The versatility of paramyxovirus RNA polymerase stuttering. J Virol 73(7):5568-5576

7. Anderson DE, Castan A, Bisaillon M, von Messling V (2012) Elements in the canine distemper virus M 3' UTR contribute to control of replication efficiency and virulence. PLoS ONE 7:e31561

8. Yamaguchi M, Kitagawa Y, Zhou M, Itoh M, Gotoh B (2014) An anti-interferon activity shared by paramyxovirus $C$ proteins: inhibition of Tolllike receptor 7/9-dependent alpha interferon induction. FEBS Lett 588:28-34

9. Fontana JM, Bankamp B, Rota PA (2008) Inhibition of interferon induction and signaling by paramyxoviruses. Immunol Rev 225:46-67

10. Goodbourn S, Randall RE (2009) The regulation of type I interferon production by paramyxoviruses. J Interf Cytokine Res 29(9):539-547

11. Parks GD, Alexander-Miller MA (2013) Paramyxovirus activation and inhibition of innate immune responses. J Mol Biol 425(24):4872-4892

12. Bernardo S, Giancotti A, Antonelli A, Rizzo G, Vinci V, Pizzuti A, Catalano C, Manganaro L (2017) MRI and US in the evaluation of fetal anomalies: the need to work together. Prenat Diagn 37:1343-1349

13. Randall RE, Goodbourn S (2008) Interferons and viruses: an interplay between induction, signalling, antiviral responses and virus countermeasures. J Gen Virol 89:1-47

14. Saito T, Hirai R, Loo YM, Owen D, Johnson CL, Sinha SC, Akira S, Fujita T Jr, Gale M (2007) Regulation of innate antiviral defenses through a shared repressor domain in RIG-I and LGP2. P Natl Acad Sci USA 104(2):582-587

15. Childs KS, Randall RE, Goodbourn S (2013) LGP2 plays a critical role in sensitizing mda-5 to activation by double-stranded RNA. PLoS ONE 8(5):e64202

16. Bruns AM, Leser GP, Lamb RA, Horvath CM (2014) The innate immune sensor LGP2 activates antiviral signaling by regulating MDA5-RNA interaction and filament assembly. Mol Cell 55(5):771-781

17. Satoh T, Kato H, Kumagai Y, Yoneyama M, Sato S, Matsushita K, Tsujimura T, Fujita T, Akira S, Takeuchi O (2010) LGP2 is a positive regulator of RIG-I- and MDA5-mediated antiviral responses. P Natl Acad Sci USA 107(4):1512-1517

18. Andrejeva J, Childs KS, Young DF, Carlos TS, Stock N, Goodbourn S, Randall RE (2004) The V proteins of paramyxoviruses bind the IFN-inducible RNA helicase, mda-5, and inhibit its activation of the IFN-beta promoter. Proc Natl Acad Sci USA 101(49): 17264-17269

19. Childs K, Stock N, Ross C, Andrejeva J, Hilton L, Skinner M, Randall R, Goodbourn S (2007) mda-5, but not RIG-I, is a common target for paramyxovirus V proteins. Virology 359(1):190-200

20. Childs KS, Andrejeva J, Randall RE, Goodbourn S (2009) Mechanism of mda-5 inhibition by paramyxovirus $\mathrm{V}$ proteins. J Virol 83(3):1465-1473

21. Davis ME, Wang MK, Rennick LJ, Full F, Gableske S, Mesman AW, Gringhuis SI, Geijtenbeet TBH, Duprex WP, Geck MU (2014) Antagonism of the phosphatase PP1 by the measles virus
$\mathrm{V}$ protein is required for innate immune escape of MDA5. Cell Host Microbe 16(1):19-30

22. Parisien JP, Bamming D, Komuro A, Ramachandran A, Rodriguez JJ, Barber G, Wojahn RD, Horvath CM (2009) A shared interface mediates paramyxovirus interference with antiviral RNA helicases MDA5 and LGP2. J Virol 83(14):7252-7260

23. Childs K, Randall R, Goodbourn S (2012) Paramyxovirus V proteins interact with the RNA Helicase LGP2 to inhibit RIGI-dependent interferon induction. J Virol 86(7):3411-3421

24. Rodriguez KR, Horvath CM (2014) Paramyxovirus V protein interaction with the antiviral sensor LGP2 disrupts MDA5 signaling enhancement but is not relevant to LGP2-mediated RLR signaling inhibition. J Virol 88(14):8180-8188

25. Ma X, Yang X, Nian X, Zhang Z, Dou T, Zhang X, Luo X, Su J, Zhu Q, Cai X (2015) Identification of amino-acid residues in the $\mathrm{V}$ protein of peste des petits ruminants essential for interference and suppression of STAT-mediated interferon signaling. Virology 483:54-63

26. Baron MD, Barrett $\mathrm{T}$ (2000) Rinderpest viruses lacking the $\mathrm{C}$ and $\mathrm{V}$ proteins show specific defects in growth and transcription of viral RNAs. J Virol 74(6):2603-2611

27. Ito M, Iwasaki M, Takeda M, Nakamura T, Yanagi Y, Ohno S (2013) Measles virus nonstructural C protein modulates viral RNA polymerase activity by interacting with host protein SHCBP1. J Virol 87(17):9633-9642

28. Nakatsu Y, Takeda M, Ohno S, Koga R, Yanagi Y (2006) Translational inhibition and increased interferon induction in cells infected with $\mathrm{C}$ protein-deficient measles virus. J Virol 80(23):11861-11867

29. Nakatsu Y, Takeda M, Ohno S, Shirogane Y, Iwasaki M, Yanagi Y (2008) Measles virus circumvents the host interferon response by different actions of the $\mathrm{C}$ and $\mathrm{V}$ proteins. J Virol 82(17):8296-8306

30. Toth AM, Devaux P, Cattaneo R, Samuel CE (2009) Protein kinase PKR mediates the apoptosis induction and growth restriction phenotypes of $\mathrm{C}$ protein-deficient measles virus. J Virol 83(2):961-968

31. McAllister CS, Toth AM, Zhang P, Devaux P, Cattaneo R, Samuel CE (2010) Mechanisms of protein kinase PKR-mediated amplification of beta interferon induction by $\mathrm{C}$ protein-deficient measles virus. J Virol 84(1):380-386

32. Takeuchi K, Komatsu T, Kitagawa Y, Sada K, Gotoh B (2008) Sendai virus $\mathrm{C}$ protein plays a role in restricting PKR activation by limiting the generation of intracellular double-stranded RNA. J Virol 82(20):10102-10110

33. Pfaller CK, Radeke MJ, Cattaneo R, Samuel CE (2014) Measles virus $C$ protein impairs production of defective copyback doublestranded viral RNA and activation of protein kinase R. J Virol 88(1):456-468

34. Pfaller CK, Mastorakos GM, Matchett WE, Ma X, Samuel CE, Cattaneo R (2015) Measles virus defective interfering RNAs are generated frequently and early in the absence of $\mathrm{C}$ protein and can be destabilized by adenosine deaminase acting on RNA-1-like hypermutations. J Virol 89(15):7735-7747

35. Sparrer KM, Pfaller CK, Conzelmann KK (2012) Measles virus C protein interferes with Beta interferon transcription in the nucleus. J Virol 86(2):796-805

36. Lasecka L, Baron M (2014) The nairovirus nairobi sheep disease virus/ganjam virus induces the translocation of protein disulphide isomerase-like oxidoreductases from the endoplasmic reticulum to the cell surface and the extracellular space. PLoS ONE 9(4):e94656

37. Devaux P, von Messling V, Songsungthong W, Springfeld C, Cattaneo R (2007) Tyrosine 110 in the measles virus phosphoprotein is required to block STAT1 phosphorylation. Virology 360:72-83 
38. Sweetman DA, Miskin J, Baron MD (2001) Rinderpest virus C and $\mathrm{V}$ proteins interact with the major $(\mathrm{L})$ component of the viral polymerase. Virology 281:193-204

39. Gotoh B, Komatsu T, Takeuchi K, Yokoo J (2001) Paramyxovirus accessory proteins as interferon antagonists. Microbiol Immunol 45:787-800

40. Horvath CM (2004) Silencing STATs: lessons from paramyxovirus interferon evasion. Cytokine Growth Factor Rev 15:117-127

41. Patterson JB, Thomas D, Lewicki H, Billeter MA, Oldstone MB (2000) V and C proteins of measles virus function as virulence factors in vivo. Virology 267:80-89

42. Bankamp B, Wilson J, Bellini WJ, Rota PA (2005) Identification of naturally occurring amino acid variations that affect the ability of the measles virus $\mathrm{C}$ protein to regulate genome replication and transcription. Virology 336:120-129

43. Boxer EL, Nanda SK, Baron MD (2009) The rinderpest virus non-structural $\mathrm{C}$ protein blocks the induction of type 1 interferon. Virology 385:134-142

44. Schuhmann KM, Pfaller CK, Conzelmann KK (2011) The measles virus $\mathrm{V}$ protein binds to $\mathrm{p} 65$ (RelA) to suppress NF-kappaB activity. J Virol 85:3162-3171
45. Shaffer JA, Bellini WJ, Rota PA (2003) The C protein of measles virus inhibits the type I interferon response. Virology 315:389-397

46. Swiech K, Picanço-Castro V, Covas DT (2012) Human cells: new platform for recombinant therapeutic protein production. Protein Expr Purif 84(1):147-153

47. Thomas P, Smart TG (2005) HEK293 cell line: a vehicle for the expression of recombinant proteins. J Pharmacol Toxicol Methods 51(3): 187-200

48. Devaux P, Cattaneo R (2004) Measles virus phosphoprotein gene products: conformational flexibility of the P/V protein aminoterminal domain and $\mathrm{C}$ protein infectivity factor function. J Virol 78(21):11632-11640

49. Takeuchi K, Ttakeda M, Miyajima N, Ami Y, Nagata N, Suzaki Y, Shahnewaz J, Kadota S, Nagata K (2005) Stringent requirement for the $\mathrm{C}$ protein of wild-type measles vires for growth both in vitro and in macaque. J Virol 79(12):7838-7844

Publisher's Note Springer Nature remains neutral with regard to jurisdictional claims in published maps and institutional affiliations. 